\title{
Longitudinal Study on the Progression of Muscle Status Among Community-Dwelling Ambulatory Older Multiethnic Asians With Type 2 Diabetes Mellitus
}

Ngiap Chuan Tan ( $\nabla$ tan.ngiap.chuan@singhealth.com.sg )

SingHealth Polyclinics

Usha Sankari

SingHealth Polyclinics

Chiat Eng Ng

SingHealth Polyclinics

Eileen Koh Yi Ling

SingHealth Polyclinics

\section{Research Article}

Keywords: muscle mass, grip strength, gait speed, type-2 diabetes mellitus, Asians

Posted Date: October 11th, 2021

DOl: https://doi.org/10.21203/rs.3.rs-900200/v1

License: (c) (1) This work is licensed under a Creative Commons Attribution 4.0 International License.

Read Full License 


\section{Abstract}

Background:

Muscle health decline with age, but its deterioration in older persons with type-2 diabetes mellitus (T2DM) over time is not well-established. This study aimed to determine the change in muscle mass, grip strength and gait speed over a period of observation among community-dwelling ambulatory older multi-ethnic Asian patients with T2DM and their associated factors.

Methods:

Among 387 eligible patients aged 60-89 years who were recruited at baseline, 348 (89.9\%) were reviewed at a public primary care clinic in Singapore in the subsequent 9 to 34 months. The change in their clinical and functional status, levels of physical activity and muscle status (mass, grip strength and gait speed based on the Asian Working Group for Sarcopenia criteria) were recorded and compared. Their physical activity levels were assessed using the Physical Activity Scale for the Elderly (PASE) and International Physical Activity Questionnaire (IPAQ). Their quality of life was evaluated based on the World Health Organization Quality of Life (WHOQOL) domains. Linear regression analysis was used to identify the factors associated with muscle health change.

Results:

The study population comprised men (52.9\%), Chinese (69.3\%), Malay (15.5\%), Indian (8.6\%), mean age of $68.4 \pm$ SD 5.6 years and had at least secondary education $(76.4 \%)$. Overall, their mean muscle mass significantly decreased by $0.7 \pm S D 1.2 \mathrm{~kg} / \mathrm{m}^{2}$, mean grip strength by $1.0 \pm S D 4.6 \mathrm{~kg}$ and negligible change in gait speed. Their mean weight significantly decreased by $0.5 \pm S D 3.9 \mathrm{~kg}$, waist and hip circumferences by $2.5 \pm S D 6 \mathrm{~cm}$ and $3.2 \pm S D 5.1 \mathrm{~cm}$ respectively, with no change in BMI. Linear regression shows significant associations between muscle mass and education level $(\beta=0.29, p=0.040,95 \% \mathrm{Cl}=0.01-0.56), \mathrm{BMI}$ $(\beta=0.10, p=0.001,95 \% \mathrm{Cl}=0.04-0.16)$ and review interval $(\beta=-0.003, p<0.001,95 \% \mathrm{Cl}=-0.004-0.002) ;$ gait speed is associated with singlehood $(\beta=-0.12, p=0.035,95 \% \mathrm{Cl}=-0.23-0.01)$ and WHOQOL physical health $(\beta=0.01, p=0.028,95 \% \mathrm{Cl}=0.00-0.02)$ domain. No factor is associated with hand grip strength change.

\section{Conclusions:}

The study population showed significant decline in their mean weight, waist and hip circumferences, mean muscle mass and mean grip strength but gait speed was unaffected. Muscle mass change was associated with education level, BMI and length of review interval. Grip strength was not correlated with any significant factor. Gait speed was associated with singlehood and physical health.

\section{Background}

Sarcopenia refers to the age-related loss of muscle mass and muscle function. The three parameters underpin muscle health of an individual. The Asian Working Group for Sarcopenia (AWGS 2014) defines 
sarcopenia as low muscle mass (defined as skeletal muscle index $<7 \mathrm{~kg} / \mathrm{m}^{2}$ in males and $<5.7 \mathrm{~kg} / \mathrm{m}^{2}$ in females) associated with either low muscle strength (defined as handgrip strength $<26 \mathrm{~kg}$ in males and $<18 \mathrm{~kg}$ in females) or low physical performance (defined as six-meter gait speed $\leq 0.8 \mathrm{~m} / \mathrm{s}$ ) or both [1].

Aging, underlying inflammation, endocrine dysfunction such as insulin resistance, nutritional deficiency and physical inactivity are risk factors for sarcopenia [2]. Insulin resistance leads to type-2 diabetes mellitus (T2DM), which is rising in prevalence in Asia, especially in regions and communities with aging populations [3]. Hence, older people with T2DM seem to be at higher risk of poor muscle health, who may be aggravated by their dietary deficiency and physical inactivity [4]. Little is known on the rate of progression of sarcopenia among these community-dwelling ambulatory older patients with T2DM [5]. A recent cross-sectional study has shown that $58 \%$ of the local older patients with T2DM had presarcopenia and sarcopenia [6]. Their risk factors such as age, diabetic nephropathy, hip circumference and multi-morbidity were significantly associated with sarcopenia. However, a single indicator of glycemic control based on the glycated hemoglobin ( $\mathrm{HbA1c}$ ) was not associated with sarcopenia in the same study. Glycemic control can be dynamic in these ambulatory older patients, being affected by their personal lifestyle and behavior such as dietary intake and physical activities over time. The longitudinal effects of combined personal demographic, clinical and environmental factors on the muscle health status of these older persons with T2DM in the community is not well-established.

In Singapore, about one in nine adults of its multi-ethnic Asian population has T2DM, with higher prevalence among its minority Malay and Indian ethnic groups. Its T2DM prevalence is estimated to increase to $15 \%$ in 2050 [7]. The World Health Organization has reported its population having the third highest longevity in the world in 2018 [8]. With increasing number of aging patients with T2DM year on year, the effect of sarcopenia on its population will escalate, with significant impact expected on their morbidity and mortality

The majority of these patients with T2DM are managed in public primary care clinics (polyclinics) on the island-state. Their continuity of care in these polyclinics present opportunities to identify risk factors associated with the progression of sarcopenia. Hence, this prospective study primarily aimed to determine the change in the muscle health status (muscle mass, grip strength and gait speed) in community dwelling, ambulatory, older multi-ethnic Asian patients with T2DM over a period of up to 34 months. The secondary aim was to identify factors which were associated with their deteriorating muscle health status over time. Understanding the progression of the muscle health and associated factors will facilitate the design of appropriate interventions to mitigate sarcopenia.

\section{Methods}

\section{Study site and period}

The baseline study was published in Fung FY et al [6]. The study data was collected at a public polyclinic in the north-eastern side of Singapore during October 2017 to March 2018. About 30\% of the clinic patient 
attendances are aged 65 years and above.

The recruited patients were reviewed and repeat clinical measurements were performed in the same polyclinic over the next 34 months. The variability in period of review was due to the COVID-19 pandemic, which disrupted regular review of patients at the study site.

\section{Study participants}

The profile of the participants included Singapore citizens or permanent residents aged 60-89 years who were enrolled into the baseline study and had a diagnosis of T2DM for at least one year in the electronic health records (EMR). The participants could be on diet control, oral hypoglycemic agents, or a combination of oral hypoglycemic agents with insulin injections.

Potential participants with history of stroke, carpal tunnel syndrome, severe hip or knee osteoarthritis, dysarthria or dysphasia, hearing difficulties, use of walking aid, physical disabilities that affected handgrip and/or walking, use of electronic implants such as pacemaker, and living in residential care facilities were excluded from the study. Those with cognitive impairment or any form of other disabilities, as documented in the EMR, which rendered them incapable of providing informed written consent, were also excluded.

\section{Sample size estimation}

Based on the study by Norshafarina SK et al [9], the prevalence of sarcopenia among seniors with T2DM is $59.8 \%$, with a $5 \%$ precision and $95 \%$ confidence level, the sample size required for the study is estimated to be 370 . A $5 \%$ buffer for incomplete data was factored in and the final sample size was 388 .

\section{Recruitment and muscle health assessment}

Potential subjects were screened for eligibility criteria at the polyclinic. They were provided information of the study using the approved documents, clarified on their queries and recruited after the research assistant obtained their informed written consent. Next, the subjects administered the study questionnaire, either by themselves or by the research assistant, to collect their demographic, lifestyle habits, socio-economic indicators and clinical information. Other information collected in the questionnaire included their level of physical activity using the validated International Physical Activity Questionnaire (IPAQ) and Physical Activity Scale for the Elderly (PASE), and their quality of life using the World Health Organization Quality of Life scale (WHOQOL) with its four domains.

\section{Questionnaire}

The IPAQ assesses the level of physical activity across a comprehensive set of domains including: leisure time physical activity, domestic and gardening (yard) activities, work-related physical activity, transportrelated physical activity. IPAQ scores are grouped according to low, moderate and high level of physical activity. If the IPAQ scores have changed from low to moderate, or moderate to high or low to high, it shows that a subject improves in level of physical activity from baseline to next time-point of 
assessment. Vice-versa, a subject who decreases in physical activity, the IPAQ has declined from moderate to low, high to moderate or high to low score. If the scores are similar, it means that subject has maintained the same physical activity level.

The PASE assesses the level of PA of community-dwelling older adults based on three domains: leisure time, household and work-related activities. PASE scores may range from zero to 400 or more, higher score indicates more time spent on physical activity. The WHOQOL 4-domain scores denote the individual's perception of quality of life in each particular domain. The 4 domains include (1) physical health, (2) psychological, (3) social relationships, and (4) environment. Higher scores denote higher quality of life.

Next, anthropometric and clinical measurements were carried out, including their weight, height, body mass index (BMI), ](WC), hip circumference (HC), systolic and diastolic blood pressures. Using the AVAMECH Model B100U device for measurement, participants can view their weight, height and BMI in a printed copy. Blood pressures were taken twice using the OMRON HEM-7280T blood pressure monitor. The blood pressures were measured twice using the automatic blood pressure monitor (OMRON HEM7280T). WC and HC were determined using the same measuring tape. WC was measured the approximate midpoint between the lower margin of the last palpable rib and the top of the iliac crest while $\mathrm{HC}$ was measured around the widest portion of the pelvis[10]. .

The definition of sarcopenia in this study was based on the AWGS 2014 diagnostic criteria[11]. The muscle health assessment was conducted in the following ways:

(1) The bio-electrical impedance analysis machine (OMRON Body composition monitor, Model HBF-375) was used to measure body muscle mass. It is then computed into skeletal muscle index by dividing the body muscle mass by squared body height.

(2) according to the American Society of Hand Therapists' guidelines, handgrip strength was measured twice on each hand, with the participant seated with elbow flexed at ninety degrees, forearm in neutral position and wrist between 0-30 degrees of dorsiflexion and supported on a Table2], using a dynamometer (JAMAR Plus Digital Hand Dynamometer \#563213). The average handgrip strength of the dominant hand was used for analysis.

(3) gait speed was computed by taking the average of two time measurements taken to walk a linear distance of 6-meters at the usual walking speed. Both run-in and run-out phases of approximately one meter were provided before and after the six-meter distance respectively. A digital stopwatch (CASIO Model 611Q24R) was used to measure the time taken.

\section{Outcomes}

Based on the AWGS criteria [10], sarcopenia is diagnosed when the skeletal muscle index is $<7 \mathrm{~kg} / \mathrm{m}^{2}$ in males and $<5.7 \mathrm{~kg} / \mathrm{m}^{2}$ in females, with either low handgrip strength of $<28 \mathrm{~kg}$ in males and $<18 \mathrm{~kg}$ in females, or low physical performance of six-meter gait speed $\leq 0.8 \mathrm{~m} / \mathrm{s}$ or both. 
The participants' latest glycemic control index (HbA1C) and fasting lipid profile (total cholesterol, highdensity lipoprotein cholesterol [HDL], low-density lipoprotein cholesterol [LDL], triglycerides [TG]) were retrieved from their laboratory test results in the electronic medical records. Other information such as the duration of T2DM, presence of diabetic complications (any documented retinopathy, nephropathy, neuropathy, vasculopathy), co-morbidities (diagnosis list) and medications (electronic prescription) were also obtained. The research assistant recorded the data in a secured research database system known as Redcap, which is password protected and accessed only by the research team members.

\section{Longitudinal data collection}

The patients were contacted by the research assistant at least 10 months later from the enrolment for repeat measurements of their anthropometry, clinical and sarcopenia related parameters at the study site. The research assistant made at least two phone calls to contact the subjects for the next review. The patients were considered dropouts if they became uncontactable or failed to show up despite the phone calls. Their latest laboratory results were again extracted from their electronic medical records during the review and documented in the Redcap research database. The latest HbA1c of the patients was compared to the glycemic control index on study enrolment. The same questionnaire was administered again to determine the change in the scores from the IPAQ, PASE and WHOQOL scales. All data from the baseline and follow up were audited, rectified and de-identified before being analyzed.

\section{Statistical analyses:}

Descriptive statistics were computed for the demographics and their physical activity and quality of life $(\mathrm{QOL})$, and the change in muscle health status were compared against the demographics using Independent t-test, ANOVA for the categorical variables, and Spearman's correlation for the continuous variables. The baseline and follow up clinical parameters were assessed using paired t-tests. The potentially significant factors ( $p<=0.2$ from bivariate analysis) affecting muscle health status were selected to be included in the multiple linear regression to control for the confounding factors. Adjusted beta estimates and their confidence intervals were presented. A p-value of less than 0.05 is considered to be statistically significant. All analyses were done using IBM SPSS version 27.0.

\section{Ethics approval and funding}

Each recruited patient received a supermarket voucher valued at SGD5 (USD3.80) at enrolment and the subsequent review. This study was approved by the SingHealth Centralized Institutional Review Board (CIRB reference 2017/2393). All methods were performed in accordance with the relevant guidelines and regulations. This study was funded by a grant from Mitsui Sumitomo Insurance Welfare Foundation. Omron Healthcare Singapore sponsored the Bio-Impedance Assessment (BIA) device.

\section{Results}

The results of the 387 patients recruited for the baseline study were published [6]. Among these patients, 39 of them failed to be contacted or defaulted the subsequent review. A total of 348 patients had repeat anthropometric and clinical measurements, constituting a study adherence rate of $89.9 \%$. The socio- 
demographic and clinical characteristics, physical activity, quality of life and anthropometry of the cohort of patients are reported in Table 1.

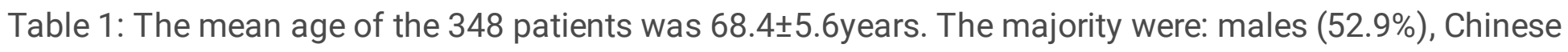
ethnicity (69.3\%), married (83.3\%), received secondary or higher education $(76.4 \%)$, live in public housing (86.8\%), live with family (95.7\%), with at least one other non-communicable disease (96.3\%), and had no change to their physical activity $(52.9 \%)$ based on their IPAQ scores during the observation period. The change in the muscle mass is significantly associated with the male gender $\left(-0.81 \mathrm{vs}-0.52 \mathrm{~kg} / \mathrm{m}^{2}\right.$ in female, $p=0.017)$, secondary or higher education level $\left(-0.76\right.$ vs $\left.-0.38 \mathrm{~kg} / \mathrm{m}^{2}, p=0.008\right)$ in those with up to primary education, social relationship $(p=0.017)$ and environment $(p=0.042)$ domains in the WHOQOL scale. With increasing length of review interval, the muscle mass further declines $(r=-0.358, p<0.001)$. Increase in $\mathrm{BMI}$ also increases the muscle mass $(r=0.153, p=0.004)$. Gait speed is significantly associated with the WHOQOL physical health domain score $(p=0.041)$. No significant factor is associated with the handgrip strength. 
Table 1

Demographics of subjects and their physical activity level

\begin{tabular}{|c|c|c|c|c|c|c|c|}
\hline & Total & $\begin{array}{l}\text { Change in } \\
\text { Muscle } \\
\text { mass }\end{array}$ & $\begin{array}{l}\text { p- } \\
\text { value }\end{array}$ & $\begin{array}{l}\text { Change } \\
\text { in HGS }\end{array}$ & $\begin{array}{l}\mathrm{p}- \\
\text { value }\end{array}$ & $\begin{array}{l}\text { Change } \\
\text { in Gait } \\
\text { speed }\end{array}$ & $\begin{array}{l}\mathrm{p}- \\
\text { value }\end{array}$ \\
\hline Total & $\begin{array}{l}348 \\
(100.0)\end{array}$ & $-0.67(1.15)$ & & $\begin{array}{l}-1.03 \\
(4.61)\end{array}$ & & $\begin{array}{l}-0.04 \\
(0.19)\end{array}$ & \\
\hline Age, years(mean/SD) & $\begin{array}{l}68.4 \\
(5.6)\end{array}$ & & 0.562 & & 0.959 & & 0.529 \\
\hline $60-69$ & $\begin{array}{l}218 \\
(62.6)\end{array}$ & $-0.64(1.16)$ & & $\begin{array}{l}-1.04 \\
(4.67)\end{array}$ & & $\begin{array}{l}-0.05 \\
(0.18)\end{array}$ & \\
\hline 70 and above & $\begin{array}{l}130 \\
(37.4)\end{array}$ & $-0.72(1.13)$ & & $\begin{array}{l}-1.01 \\
(4.53)\end{array}$ & & $\begin{array}{l}-0.03 \\
(0.2)\end{array}$ & \\
\hline Gender & & & 0.017 & & 0.331 & & 0.939 \\
\hline Male & $\begin{array}{l}184 \\
(52.9)\end{array}$ & $-0.81(1.15)$ & & $\begin{array}{l}-1.26 \\
(5.4)\end{array}$ & & $\begin{array}{l}-0.04 \\
(0.19)\end{array}$ & \\
\hline Female & $\begin{array}{l}164 \\
(47.1)\end{array}$ & $-0.52(1.14)$ & & $\begin{array}{l}-0.77 \\
(3.52)\end{array}$ & & $\begin{array}{l}-0.04 \\
(0.19)\end{array}$ & \\
\hline Ethnicity & & & 0.630 & & 0.958 & & 0.500 \\
\hline Chinese & $\begin{array}{l}241 \\
(69.3)\end{array}$ & $-0.65(1.06)$ & & $\begin{array}{l}-0.96 \\
(4.68)\end{array}$ & & $\begin{array}{l}-0.03 \\
(0.19)\end{array}$ & \\
\hline Malay & $\begin{array}{l}54 \\
(15.5)\end{array}$ & $-0.84(1.44)$ & & $\begin{array}{l}-1.03 \\
(4.52)\end{array}$ & & $\begin{array}{l}-0.05 \\
(0.19)\end{array}$ & \\
\hline Indian & $\begin{array}{l}30 \\
(8.6)\end{array}$ & $-0.64(1.35)$ & & $\begin{array}{l}-1.39 \\
(4.25)\end{array}$ & & $\begin{array}{l}-0.03 \\
(0.16)\end{array}$ & \\
\hline Others & $\begin{array}{l}23 \\
(6.6)\end{array}$ & $-0.51(1.11)$ & & $\begin{array}{l}-1.28 \\
(4.81)\end{array}$ & & $\begin{array}{l}-0.1 \\
(0.18)\end{array}$ & \\
\hline Marital status & & & 0.333 & & 0.360 & & 0.140 \\
\hline Married & $\begin{array}{l}290 \\
(83.3)\end{array}$ & $-0.7(1.2)$ & & $\begin{array}{l}-0.86 \\
(4.85)\end{array}$ & & $\begin{array}{l}-0.04 \\
(0.18)\end{array}$ & \\
\hline Single & $\begin{array}{l}11 \\
(3.2)\end{array}$ & $-0.68(1.04)$ & & $\begin{array}{l}-0.87 \\
(2.14)\end{array}$ & & $\begin{array}{l}-0.15 \\
(0.17)\end{array}$ & \\
\hline Divorced/Separated & $14(4)$ & $-0.91(0.88)$ & & $\begin{array}{l}-1.85 \\
(4.26)\end{array}$ & & $\begin{array}{l}0.01 \\
(0.25)\end{array}$ & \\
\hline
\end{tabular}

*PASE-Physical Activity Scale for the Elderly

*WHOQOL-World Health Organization Quality of Life scale: Domain 1-Physical health, Domain 2-

Psychological, Domain 3-Social relationship, Domain 4-Environment

*IPAQ - International Physical Activity Questionnaire. 


\begin{tabular}{|c|c|c|c|c|c|c|c|}
\hline & Total & $\begin{array}{l}\text { Change in } \\
\text { Muscle } \\
\text { mass }\end{array}$ & $\begin{array}{l}\mathrm{p}- \\
\text { value }\end{array}$ & $\begin{array}{l}\text { Change } \\
\text { in HGS }\end{array}$ & $\begin{array}{l}\mathrm{p}- \\
\text { value }\end{array}$ & $\begin{array}{l}\text { Change } \\
\text { in Gait } \\
\text { speed }\end{array}$ & $\begin{array}{l}\mathrm{p} \text { - } \\
\text { value }\end{array}$ \\
\hline Widowed & $\begin{array}{l}33 \\
(9.5)\end{array}$ & $-0.34(0.81)$ & & $\begin{array}{l}-2.26 \\
(2.75)\end{array}$ & & $\begin{array}{l}-0.02 \\
(0.22)\end{array}$ & \\
\hline Highest Qualification & & & 0.008 & & 0.156 & & 0.634 \\
\hline $\begin{array}{l}\text { Up to primary } \\
\text { education }\end{array}$ & $\begin{array}{l}82 \\
(23.6)\end{array}$ & $-0.38(1.18)$ & & $\begin{array}{l}-0.4 \\
(4.65)\end{array}$ & & $\begin{array}{l}-0.03 \\
(0.19)\end{array}$ & \\
\hline $\begin{array}{l}\text { Secondary education } \\
\text { and beyond }\end{array}$ & $\begin{array}{l}266 \\
(76.4)\end{array}$ & $-0.76(1.13)$ & & $\begin{array}{l}-1.23 \\
(4.59)\end{array}$ & & $\begin{array}{l}-0.04 \\
(0.19)\end{array}$ & \\
\hline Type of dwelling & & & 0.541 & & 0.790 & & 0.595 \\
\hline $\begin{array}{l}\text { Public housing (Rental } \\
\text { flat/1-3room) }\end{array}$ & $\begin{array}{l}43 \\
(12.4)\end{array}$ & $-0.13(-2.66)$ & & $\begin{array}{l}0.17 \\
(-8.05)\end{array}$ & & $\begin{array}{l}-0.01 \\
(-0.41)\end{array}$ & \\
\hline $\begin{array}{l}\text { Public housing (4-5 } \\
\text { room) }\end{array}$ & $\begin{array}{l}259 \\
(74.4)\end{array}$ & $-0.56(-3.74)$ & & $\begin{array}{l}-0.54 \\
(-20.85)\end{array}$ & & $\begin{array}{l}-0.02 \\
(-0.67)\end{array}$ & \\
\hline $\begin{array}{l}\text { Condominium/Private } \\
\text { property }\end{array}$ & $\begin{array}{l}46 \\
(13.2)\end{array}$ & $-0.29(-5.76)$ & & $\begin{array}{l}0.76 \\
(-13.8)\end{array}$ & & $\begin{array}{l}0.03 \\
(-0.4)\end{array}$ & \\
\hline Living & & & 0.837 & & 0.150 & & 0.615 \\
\hline Alone & $\begin{array}{l}15 \\
(4.3)\end{array}$ & $-0.73(0.82)$ & & $\begin{array}{l}-2.71 \\
(4.28)\end{array}$ & & $\begin{array}{l}-0.07 \\
(0.18)\end{array}$ & \\
\hline $\begin{array}{l}\text { With family/ domestic } \\
\text { helper }\end{array}$ & $\begin{array}{l}333 \\
(95.7)\end{array}$ & $-0.67(1.16)$ & & $\begin{array}{l}-0.95 \\
(4.62)\end{array}$ & & $\begin{array}{l}-0.04 \\
(0.19)\end{array}$ & \\
\hline \multicolumn{8}{|l|}{ Comorbidities } \\
\hline $\begin{array}{l}\text { Hypertension/ High } \\
\text { blood pressure }\end{array}$ & & & 0.756 & & 0.262 & & 0.367 \\
\hline Yes & $\begin{array}{l}302 \\
(86.8)\end{array}$ & $-0.66(1.14)$ & & $\begin{array}{l}-1.14 \\
(4.7)\end{array}$ & & $\begin{array}{l}-0.04 \\
(0.19)\end{array}$ & \\
\hline No & $\begin{array}{l}46 \\
(13.2)\end{array}$ & $-0.72(1.21)$ & & $\begin{array}{l}-0.32 \\
(3.97)\end{array}$ & & $\begin{array}{l}-0.02 \\
(0.19)\end{array}$ & \\
\hline $\begin{array}{l}\text { Hyperlipidemia/ High } \\
\text { Cholesterol }\end{array}$ & & & 0.805 & & 0.814 & & 0.557 \\
\hline Yes & $\begin{array}{l}335 \\
(96.3)\end{array}$ & $-0.67(1.16)$ & & $\begin{array}{l}-1.04 \\
(4.64)\end{array}$ & & $\begin{array}{l}-0.04 \\
(0.19)\end{array}$ & \\
\hline
\end{tabular}

* PASE-Physical Activity Scale for the Elderly

*WHOQOL-World Health Organization Quality of Life scale: Domain 1-Physical health, Domain 2-

Psychological, Domain 3- Social relationship, Domain 4-Environment

*IPAQ - International Physical Activity Questionnaire. 


\begin{tabular}{|c|c|c|c|c|c|c|c|}
\hline & Total & $\begin{array}{l}\text { Change in } \\
\text { Muscle } \\
\text { mass }\end{array}$ & $\begin{array}{l}\text { p- } \\
\text { value }\end{array}$ & $\begin{array}{l}\text { Change } \\
\text { in HGS }\end{array}$ & $\begin{array}{l}\text { p- } \\
\text { value }\end{array}$ & $\begin{array}{l}\text { Change } \\
\text { in Gait } \\
\text { speed }\end{array}$ & $\begin{array}{l}\text { p- } \\
\text { value }\end{array}$ \\
\hline No & $\begin{array}{l}13 \\
(3.7)\end{array}$ & $-0.59(0.93)$ & & $\begin{array}{l}-0.73 \\
(4.03)\end{array}$ & & $\begin{array}{l}-0.01 \\
(0.16)\end{array}$ & \\
\hline Ischemic Heart Disease & & & 0.553 & & 0.589 & & 0.761 \\
\hline Yes & $\begin{array}{l}64 \\
(18.4)\end{array}$ & $-0.75(1.1)$ & & $\begin{array}{l}-0.75 \\
(5.48)\end{array}$ & & $\begin{array}{l}-0.03 \\
(0.19)\end{array}$ & \\
\hline No & $\begin{array}{l}283 \\
(81.6)\end{array}$ & $-0.66(1.16)$ & & $\begin{array}{l}-1.1 \\
(4.4)\end{array}$ & & $\begin{array}{l}-0.04 \\
(0.19)\end{array}$ & \\
\hline $\begin{array}{l}\text { Total number of } \\
\text { medical conditions }\end{array}$ & $\begin{array}{l}4.5 \\
(1.6)\end{array}$ & -0.038 & 0.480 & -0.030 & 0.584 & 0.009 & 0.873 \\
\hline $\begin{array}{l}\text { Total number of long } \\
\text { term medications }\end{array}$ & $\begin{array}{l}6.1 \\
(2.5)\end{array}$ & -0.045 & 0.403 & -0.014 & 0.793 & -0.009 & 0.869 \\
\hline $\begin{array}{l}\text { Follow up period } \\
\text { (days) }\end{array}$ & $\begin{array}{l}560 \\
(122)\end{array}$ & -0.358 & $<0.001$ & 0.064 & 0.237 & -0.101 & 0.060 \\
\hline \multicolumn{8}{|l|}{ Change from Baseline } \\
\hline BMI & $0(1.9)$ & 0.153 & 0.004 & 0.039 & 0.472 & 0.002 & 0.964 \\
\hline HbA1c & $-0.1(1)$ & 0.057 & 0.298 & 0.100 & 0.066 & 0.008 & 0.888 \\
\hline PASE, Mean (SD) & $\begin{array}{l}0.4 \\
(88.4)\end{array}$ & -0.045 & 0.408 & 0.088 & 0.105 & -0.050 & 0.352 \\
\hline \multicolumn{8}{|l|}{ WHOQOL } \\
\hline Domain 1 & $\begin{array}{l}0.2 \\
(1.8)\end{array}$ & 0.066 & 0.218 & 0.005 & 0.932 & 0.11 & 0.041 \\
\hline Domain 2 & $\begin{array}{l}0.9 \\
(1.8)\end{array}$ & -0.013 & 0.806 & 0.062 & 0.247 & 0.018 & 0.744 \\
\hline Domain 3 & $\begin{array}{l}2.2 \\
(3.2)\end{array}$ & 0.128 & 0.017 & -0.021 & 0.701 & 0.034 & 0.524 \\
\hline Domain 4 & $\begin{array}{l}-0.5 \\
(2.2)\end{array}$ & 0.109 & 0.042 & 0.020 & 0.714 & -0.027 & 0.621 \\
\hline IPAQ & & & 0.260 & & 0.576 & & 0.668 \\
\hline No change & $\begin{array}{l}175 \\
(52.9)\end{array}$ & $-0.7(1.2)$ & & $\begin{array}{l}-0.9 \\
(3.8)\end{array}$ & & $0(0.2)$ & \\
\hline \multicolumn{8}{|c|}{ *PASE-Physical Activity Scale for the Elderly } \\
\hline \multicolumn{8}{|c|}{$\begin{array}{l}\text { * WHOQOL-World Health Organization Quality of Life scale: Domain 1- Physical health, Domain 2- } \\
\text { Psychological, Domain 3- Social relationship, Domain 4- Environment }\end{array}$} \\
\hline *IPAQ - International I & al $A c$ & Questic & & & & & \\
\hline
\end{tabular}




\begin{tabular}{|c|c|c|c|c|c|c|c|}
\hline & Total & $\begin{array}{l}\text { Change in } \\
\text { Muscle } \\
\text { mass }\end{array}$ & $\begin{array}{l}\mathrm{p}- \\
\text { value }\end{array}$ & $\begin{array}{l}\text { Change } \\
\text { in HGS }\end{array}$ & $\begin{array}{l}\text { p- } \\
\text { value }\end{array}$ & $\begin{array}{l}\text { Change } \\
\text { in Gait } \\
\text { speed }\end{array}$ & $\begin{array}{l}\mathrm{p}- \\
\text { value }\end{array}$ \\
\hline Improve & $\begin{array}{l}68 \\
(20.5)\end{array}$ & $-0.6(1.1)$ & & $\begin{array}{l}-1.6 \\
(4.3)\end{array}$ & & $-0.1(0.2)$ & \\
\hline Worse & $\begin{array}{l}88 \\
(26.6)\end{array}$ & $-0.9(1)$ & & $-1.2(6)$ & & $0(0.2)$ & \\
\hline \multicolumn{8}{|c|}{ * PASE-Physical Activity Scale for the Elderly } \\
\hline \multicolumn{8}{|c|}{$\begin{array}{l}\text { * WHOQOL-World Health Organization Quality of Life scale: Domain 1-Physical health, Domain 2- } \\
\text { Psychological, Domain 3- Social relationship, Domain 4-Environment }\end{array}$} \\
\hline
\end{tabular}

Table 2: The following clinical parameters are significantly reduced over the observation period: weight $(-0.49 \pm 3.9 \mathrm{~kg}, \mathrm{p}<0.02)$, waist circumference $(-2.48 \pm 5.96 \mathrm{~cm}, p<0.001)$, hip circumference $(-3.18 \pm 5.09 \mathrm{~cm}$, $p<0.001)$, systolic blood pressure $(-5.27 \pm 19.26 \mathrm{mmHg}, p<0.001)$, diastolic blood pressure $(-2.71 \pm 10.08 \mathrm{mmHg}, p<0.001)$, HBA1c $(-0.13 \pm 1.0 \%, p=0.016)$, muscle mass $\left(-0.67 \pm 1.15 \mathrm{~kg} / \mathrm{m}^{2}, p<0.001\right)$ and hand grip strength $(-0.58 \pm 7.76 \mathrm{~kg}, \mathrm{p}<0.001)$. No significant change is noted in their gait speed and mean PASE score. Their quality of life scores significantly increase in the psychological, social relationship and environmental domains in the WHOQOL scale.

Table 2. Change in clinical parameters between baseline and follow up visit 


\begin{tabular}{|c|c|c|c|c|}
\hline & Baseline & Follow up & $\begin{array}{l}\text { p- } \\
\text { value }\end{array}$ & $\begin{array}{l}\text { Change from Baseline } \\
\text { (SD) }\end{array}$ \\
\hline Weight (kg) & $65.7(12.4)$ & $65.3(12.2)$ & 0.020 & $-0.49(3.9)$ \\
\hline $\mathrm{BMI}\left(\mathrm{kg} / \mathrm{m}^{2}\right)$ & $25.4(4.1)$ & $25.4(4.2)$ & 0.767 & $-0.03(1.89)$ \\
\hline Waist circumference (cm) & $94.7(10.3)$ & $92.3(10.2)$ & $<0.001$ & $-2.48(5.96)$ \\
\hline Hip circumference (cm) & $102.4(8.1)$ & $99.2(8.1)$ & $<0.001$ & $-3.18(5.09)$ \\
\hline Systolic BP (mmHg) & $\begin{array}{l}140.8 \\
(17.9)\end{array}$ & $135.5(17)$ & $<0.001$ & $-5.27(19.26)$ \\
\hline Diastolic BP (mmHg) & $77.9(9.8)$ & $75.2(9.7)$ & $<0.001$ & $-2.71(10.08)$ \\
\hline $\begin{array}{l}\text { HbA1c (\%)HBA1c } \\
(-0.13+1.0 \%\end{array}$ & $7.2(1)$ & $7.1(1.2)$ & 0.016 & $-0.13(1.00)$ \\
\hline Muscle Mass $\left(\mathrm{kg} / \mathrm{m}^{2}\right)$ & $6.3(1.3)$ & $5.6(1.5)$ & $<0.001$ & $-0.67(1.15)$ \\
\hline Hand Grip strength (kg) & $25.6(8.1)$ & $24.6(8.1)$ & $<0.001$ & $-0.58(7.76)$ \\
\hline Gait Speed (m/sec) & $1.02(0.17)$ & $0.98(0.2)$ & $<0.001$ & $-0.04(0.19)$ \\
\hline PASE, Mean (SD) & $121(80.6)$ & $\begin{array}{l}121.8 \\
(82.8)\end{array}$ & 0.893 & $0.36(88.45)$ \\
\hline \multicolumn{5}{|l|}{ WHOQOL* } \\
\hline Domain 1 & $14.8(1.7)$ & $15(1.4)$ & 0.192 & $0.15(1.82)$ \\
\hline Domain 2 & $15.3(1.8)$ & $16.2(1.3)$ & $<0.001$ & $0.91(1.85)$ \\
\hline Domain 3 & $12.8(3.1)$ & $14.4(2.4)$ & $<0.001$ & $2.20(3.19)$ \\
\hline Domain 4 & $15.4(1.9)$ & $15.9(1.1)$ & $<0.001$ & $-0.48(2.16)$ \\
\hline \multicolumn{5}{|c|}{ * PASE-Physical Activity Scale for the Elderly } \\
\hline \multicolumn{5}{|c|}{$\begin{array}{l}\text { *WHOQOL- World Health Organization Quality of Life scale: Domain 1- Physical health, Domain 2- } \\
\text { Psychological, Domain 3-Social relationship, Domain 4-Environment }\end{array}$} \\
\hline \multicolumn{5}{|c|}{$\begin{array}{l}\text { Table 3: With the potential factors ( } p \text {-values }<=0.2) \text { included in the linear regression, the results show that } \\
\text { lower education }(p=0.040) \text {, shorter length of review interval }(p<0.001) \text { and increase in } B M I(p=0.001) \text { are } \\
\text { associated with increase of muscle mass; singlehood }(p=0.033) \text { and decline in the physical health } \\
(p=0.038) \text { based on WHOQOL scale are associated with gait speed reduction. }\end{array}$} \\
\hline
\end{tabular}




\begin{tabular}{|c|c|c|}
\hline Change in Muscle mass & Beta $(95 \% \mathrm{Cl})$ & p-value \\
\hline \multicolumn{3}{|l|}{ Gender } \\
\hline Male & $-0.19(-0.42-0.04)$ & 0.109 \\
\hline Female & Ref & - \\
\hline \multicolumn{3}{|l|}{ Highest Qualification } \\
\hline Up to primary education & $0.29(0.01-0.56)$ & 0.040 \\
\hline Secondary education and beyond & Ref & - \\
\hline \multicolumn{3}{|l|}{ WHOQOL } \\
\hline Domain 3 & $0.01(-0.03-0.05)$ & 0.667 \\
\hline Domain 4 & $0.04(-0.02-0.1)$ & 0.164 \\
\hline Follow up period (days) & $-0.003(-0.004-0.002)$ & $<0.001$ \\
\hline Change in BMI & $0.10(0.04-0.16)$ & 0.001 \\
\hline Change in hand grip strength & Beta $(95 \% \mathrm{Cl})$ & p-value \\
\hline \multicolumn{3}{|l|}{ Highest Qualification } \\
\hline Up to primary education & $1.04(-0.11-2.18)$ & 0.075 \\
\hline Secondary education and beyond & Ref & - \\
\hline \multicolumn{3}{|l|}{ Living } \\
\hline Alone & $-1.8(-4.13-0.52)$ & 0.129 \\
\hline With family/ Domestic helper & Ref & \\
\hline Change in $\mathrm{HbA1c}$ & $0.48(0-0.97)$ & 0.051 \\
\hline Change in PASE & $0(0-0.01)$ & 0.188 \\
\hline Change in Gait speed & Beta $(95 \% \mathrm{Cl})$ & p-value \\
\hline \multicolumn{3}{|l|}{ Marital status } \\
\hline Married & Ref & \\
\hline Single & $-0.12(-0.23-0.01)$ & 0.035 \\
\hline Divorced/Separated & $0.06(-0.05-0.16)$ & 0.281 \\
\hline Widowed & $0.02(-0.05-0.09)$ & 0.550 \\
\hline \multicolumn{3}{|l|}{ WHOQOL } \\
\hline Domain 1 & $0.01(0.00-0.02)$ & 0.030 \\
\hline
\end{tabular}



Follow up period (days)
$0.00(0.00-0.00)$
0.530

* WHOQOL-World Health Organization Quality of Life scale: Domain 1- Physical health, Domain 2Psychological, Domain 3- Social relationship, Domain 4-Environment

\section{Discussion}

The study tracks the anthropometric and muscle health parameters, together with self-reported physical activity and quality of life outcomes of older Asian patients with T2DM longitudinally over a period of up to 34 months. Hyperglycemia is a risk factor associated with sarcopenia compared with those with normoglycemia [12]. This study population of older patients had relatively stable glycemia and blood pressures based on their improved clinical outcomes in $\mathrm{HbA1c}$, systolic and diastolic blood pressure indices (Table 1). Nonetheless, their muscle health parameters including muscle mass, strength and gait speed declined over the study period despite optimal control of their medical conditions and increased quality of life based on the WHOQOL scores (Table 2). The exception is the insignificant marginal QOL change in the WHOQOL physical health domain, which may indirectly reflect on the consequences of their gradual decline in muscle health (Table 2). Correspondingly, their weight, waist and hip circumferences decreased even though their self-reported physical activity level based on the PASE scores remained largely similar (Table2). Overall, the results suggest regression of muscle health across the three major parameters over time in older patients with T2DM despite stable medical health status.

Males showed higher reduction in muscle mass compared with their female counterparts (Tables 1) but gender becomes an insignificant factor after linear regression analysis (Table 3a). Several studies have revealed a male preponderance towards sarcopenia based on AWGS criteria [13]. However, the study populations in these studies were heterogenous, comprising healthy volunteers or those with and without T2DM. Longitudinal analysis by Shimokata $\mathrm{H}$ et al had shown that the skeletal muscle mass decreased with aging over a 12-year period except in Japanese middle-aged and elderly men [14]. However only $6.5 \%$ of men and $3.8 \%$ of women in this study population had T2DM. While insulin resistance is a risk factor associated with sarcopenia, its effect cuts across both gender in this study population with T2DM. Furthermore, the glycemic control remained stable over the study period.

Tables 1 and 3: The muscle mass of patients with lower education declined at a smaller magnitude compared to those with higher education level. Education was not associated with muscle strength and performance (Table 3). In contrast, the InCHIANTI study led by S Volpato et al revealed that years of education were associated with reduced likelihood of sarcopenia in community dwelling older Italian people [15]. The years of education is a surrogate indicator for educational level, but with caveats. They also alluded to educational level being specifically correlated with muscle strength and walking speed rather than muscle mass alone. Nonetheless, they reported that their cross-sectional and observational design of the study limited any temporal or cause-effect relationship between muscle health parameters and associated factors. The older patients with lower education could have different lifestyle beyond 
physical activity. Potential confounding factors such as diet and nutrition status, were not recorded in this study, which constitute a limitation [16].

Table 3: Muscle mass decreases with time, which is compatible with ageing. It declines by an estimated mean of $0.003 \mathrm{~kg} /$ day or about $1 \mathrm{~kg}$ per year, alluding to a need for a review of muscle mass annually for any older person. Alternatively muscle mass increases by $0.1 \mathrm{~kg}$ with every unit increase in BMI (Table 3 ). $\mathrm{BMI}$ can be a surrogate marker for muscle mass if bio-impedance assessment is unavailable, assuming no other confounders on the BMI.

No significant demographic and clinical factor was associated with the change in grip strength. Hence, general evidence-based interventions can potentially alleviate the decline in muscle strength in older patients with T2DM, without targeting any specific risk factors. Muscle strength is a risk factor for adverse outcomes, such as falls, functional decline, cardiovascular disease and mortality in older people [17]. Its measurement is pivotal to determine the muscle health of any older person. However, monitoring of muscle strength based on grip strength using a dynamometer is not routinely conducted in primary care, as the device is often not available in most general practice clinics. The Singapore Multidisciplinary Consensus on Muscle Health recommends an alternative method of muscle strength by recording the timing to complete the 5-times Chair-Stand test [18]. The recommended cut-off is 8.5 seconds, which can be easily measured by a watch in a clinical setting. Such serial measurements of muscle strength can be used to motivate any older adult to take on suitable interventions to sustain their muscle health.

Muscle function or performance, as reflected in the gait speed, was significantly associated with physical health domain in the WHOQOL scale (Table 3). Mobility is determined by muscle performance [19]. In a local study of older people, mobility is the second commonest factor to impact on their quality of life [20]. Adequate muscle function enables the older people to ambulate and venture out of their residence to carry out their exercises and physical activities in the community. Aside from improving their physical health, their quality of life is also enhanced.

Singlehood is associated with lower muscle performance (Table 3). While the single older adult may live with other siblings in the same household, a proportion of them tend to stay alone or with a non-family room-mate due to local housing policy. In 2020, there were approximately 63.8 thousand single-person elderly households in Singapore [21]. Linton et al have reported that older adults who live alone generally have comparable overall financial adequacy, physical and social well-being vis-à-vis to those who are in other living arrangements [22]. Nevertheless, a higher percentage of older adults living alone report having depressive symptoms, but it is uncertain if their symptoms are attributed entirely to their living arrangements [23]. Physical inactivity is associated with depressive symptoms, which may be detrimental to their muscle function. However, Linton et al also reported that these depressive symptoms were moderated by the extent of the older persons' social network. The authors also highlighted that the feeling of loneliness rather than the living arrangement per se which is associated with worse health outcome. Further research is needed to tease out the complex relationship between single older patients with T2DM and their muscle performance. 
The PASE scores show insignificant change in physical activity among the older patients over the study period (Table 1). Their IPAQ scores showed that $52.9 \%$ of the study population had no change and $26.6 \%$ of them had decreased physical activities (Table 1). A local recent survey revealed similar physical activity profile of older adults with a median PASE score of 110 , of which $37 \%$ of them spending eight or more sedentary hours daily [24]. Interventions are deemed necessary to strengthen their muscle health whilst keeping their glycaemia and other medical conditions under control. The Singapore Multidisciplinary Consensus Recommendations on Muscle Health suggests that for the uncomplicated cases, lifestyle modifications in exercise and diet can be initiated in the community without further assessment [18]. However, during the current COVID-19 pandemic, official enforcement measures such as isolation, quarantine and social distancing result in extended period of time at home. Consequently, reductions in physical activity and changes to dietary intake could potentially adversely affect the muscle health of these older people [25]. Home-based exercise is a potential solution to deter sedentary behavior and physical inactivity during the pandemic in older adults, especially those with T2DM in the study [26]. Suitable home-based physical activity and exercises will not only support their muscle health but also their glycemic control and mental health.

\section{Strength}

This longitudinal study is one of the few which focuses on the short term clinical examination of muscle mass, strength and function in older patients with T2DM. The results show deterioration of muscle health despite stable glycemic control in this cohort of community dwelling Asian patients. Almost $90 \%$ of the study population had completed the study despite the current Covid-19 pandemic.

\section{Limitations}

The study has its limitations. The framework for the muscle health parameters is based on the AWGS 2014 guidelines as the study was initiated in 2017 and continued till early 2021. The skeletal muscle mass is used as a major parameter instead of the appendicular muscle mass due to resources available for the study in 2017. The physical activity assessment is self-reported in the PASE and IPAQ scales, which is another limitation. Other factors such as dietary intake and nutritional status are not recorded in this study, which may confound the results.

\section{Conclusion}

Despite stable glycemic control, older multi-ethnic Asian patients with T2DM had significant decline in their mean weight, waist and hip circumference, mean muscle mass and mean grip strength but no significant change in gait speed over a period of up to 34 months. Muscle mass change was associated with education level, BMI and length of review interval. No significant factor was associated with grip strength. Gait speed was associated with singlehood and WHOQOL physical health domain. Preventive measures such as home-based physical activity may be a potential solution for this group of vulnerable patients, especially amidst the Covid-19 pandemic, but further research is needed to validate this option in strengthening their muscles. 


\section{Abbreviations}

\begin{tabular}{|ll|}
\hline AWGS & Asian Working Group for Sarcopenia \\
\hline BMI & Body Mass Index \\
\hline CI & Confidence Interval \\
\hline CIRB & Centralized Institutional Review Board \\
\hline DEXA & Dual-energy X-ray Absorptiometry \\
\hline EMR & Electronic Medical Records \\
\hline EWGSOP & European Working Group on Sarcopenia in Older People \\
\hline HBA1c & Hemoglobin A1c/ Glycated Hemoglobin \\
\hline HC & Hip Circumference \\
\hline HDL & High-density Lipoprotein cholesterol \\
\hline LDL & Low-density Lipoprotein cholesterol \\
\hline NCD & Non-communicable disease \\
\hline NEFA & Non-esterified Fatty Acids \\
\hline OR & Odds Ratio \\
\hline SD & Standard Deviation \\
\hline SPSS & Statistical Package for Social Science \\
\hline T2DM & Type 2 Diabetes Mellitus \\
\hline TG & Triglycerides \\
\hline WC & Waist Circumference \\
\hline WHO & World Health Organization \\
\hline
\end{tabular}

\section{Declarations}

Ethics approval and consent to participate in the study:

This study was reviewed and approved by the SingHealth Centralized Institutional Review Board (CIRB reference 2017/2393). Participants were provided study related information using the approved patient information sheet. Their queries were addressed and enrolled to the study after the investigator obtained their written informed consent.

Consent for publication: Not applicable 
Availability of data and material: The datasets are not available from the corresponding author due to restrictions based on the protocol approval by the institution review board.

Competing Interests: The authors declare that they have no competing interests.

Funding:

This study was funded by a grant from Mitsui Sumitomo Insurance Welfare Foundation. The Omron Healthcare Singapore sponsored the Bio-impedance Assessment (BIA) device. The funding bodies had no role in the design of the study and collection, analysis, and interpretation of data and in writing the manuscript.

Authors' contributions

TNC designed the longitudinal study protocol. US audited the data and organized it for analysis. KYLE analyzed the data. KYLE \& TNC interpreted and reported the results. TNC drafted the manuscript. Both TNC and KYLE reviewed and finalized the manuscript.

Acknowledgements

The authors are grateful to Mitsui Sumitomo Insurance Welfare Foundation Research Grant for the main sponsorship of the study and Omron Healthcare Singapore Private Limited for sponsoring the BIA devices to conduct the muscle mass assessment. They are thankful to Caris Tan and Patricia Kin for their administrative support to obtain CIRB approval and procurement of vouchers and consumables.

\section{References}

1. Chen L, Liu L, Woo J, Assantachai P, Auyeung T, et al. Sarcopenia in Asia: Consensus Report of the Asian Working Group for Sarcopenia. JAMDA 15, 2014;95-101.

2. Cruz-Jentoft AJ, Baeyens JP, Bauer JM, Boirie Y, Cederholm T, Landi F, et al. Sarcopenia: European consensus on definition and diagnosis. Age Ageing. 2010;39:412-23.

3. Ramachandran A. Trends in prevalence of diabetes in Asian countries. World J Diabetes. 2012;3:110. doi:10.4239/wjd.v3.i6.110.

4. Muhammad A Abdul-Ghani, ralph A DeFronzo. Pathogenesis of Insulin Resistance in Skeletal Muscle. Biomed Research International 2010. Https://doi.org/10.1155/2010/476279.

5. S. Kaushik, R. Singh and A.M. Cuervo. Autophagic pathways and metabolic stress. Diabetes Obes Metab. 2010;October 12(0 2):4-14. Doi: 10.1111/j.1463-1326.2010.01263.x

6. Fung FY, Koh YLE, Malhothra R, Ostbye T, Lee PY, Shariff Ghazali S, Tan NC. Prevalence of and factors associated with sarcopenia among multi-ethnic ambulatory older Asians with type-2 diabetes mellitus in a primary care setting. BMC Geriatr.2019 Apr 29;18(1):122. doi: 10.1186/s12877-0191137-8. PMID:31035928; PMCID: PMC6489356. 
7. Phan TP, Alkema L, Tai ES, Tan KHX, Yang Q, Lim W-Y, et al. Forecasting the burden of type 2 diabetes in Singapore using a demographic epidemiological model of Singapore. BMJ Open Diabetes Res Care. 2014;2:e000012. doi:10.1136/bmjdrc-2013-000012.

8. Life Expectancy. Global Health Observatory (GHO) data. http://www.who.int/gho/mortality_burden_disease/life_tables/situation_trends/en/.

9. Norshafarina SK, Noor Ibrahim MS, Suzana S, Mohamad Hasnan A, Zahara M, Zaitun Y. Sarcopenia and its impact on health: Do they have significant associations? Sains Malaysiana. 2013;42:134555 .

10. Waist circumference and waist-hip ratio: Report of a WHO Expert Consultation Geneva, 8-11 December 2008.

11. Chen LK, Liu LK, Woo J, et al. Sarcopenia in Asia: Consensus Report of the Asian Working Group for Sarcopenia. JAMDA 15 (2014) 95-101.http://dx.doi.org/10.1016/j.jamda.2013.11.025

12. Leenders $M$, Verdijk LB, van der Hoeven $L$, et al. Patients with type 2 diabetes patients show a greater decline in muscle mass, muscle strength, and functional capacity with aging. J Am Med Dir Assoc. 2013;14:585-92.

13. Du Y, Wang $X, X i e ~ H$ et al. Sex differences in the prevalence and adverse outcomes of sarcopenia and sarcopenic obesity in community dwelling elderly in East China using the AWGS criteria. BMC Endocrine Disorders (2019) 19:109 https://doi.org/10.1186/s12902-019-0432-x.

14. Shimokata $H$, Ando F, Yuki A, Otsuka R. Age-related changes in skeletal muscle mass among community-dwelling Japanese: a 12-year longitudinal study. Geriatr Gerontol Int. 2014;14(Suppl 1):85-92.

15. S. Volpato, L. Bianchi, A. Cherubini, F. Landi, M. Maggio, E. Savino, S. Bandinelli, G.P. Ceda, J.M. Guralnik, G. Zuliani, L. Ferrucci, Prevalence and clinical correlates of sarcopenia in communitydwelling older people: application of the EWGSOP definition and diagnostic algorithm, The journals of gerontology. Series A, Biological sciences and medical sciences 69(4) (2014) 438-446.

16. Siew Ling Tey, Samuel Teong Huang Chew, Choong How How, Menaka Yalawar et al. Factors associated with muscle mass in community-dwelling older people in Singapore: Findings from the SHIELD Study. PLOS One. 2019 Oct 9:14(10):e0223222.

Doi:10.1371/journal.pone.0223222.eCollection 2019.

17. Papadopoulou SK. Sarcopenia: A Contemporary Health Problem among Older Adult Populations. Nutrients. 2020;12(5):1293. Published 2020 May 1. doi:10.3390/nu12051293.

18. Chew STH, Kayambu G, Lew CCH, Ng TP, Ong F, Tan J, Tan NC, Tham SL. Singapore multidisciplinary consensus recommendations on muscle health in older adults: assessment and multimodal targeted intervention across the continuum of care. BMC Geriatr. 2021 May 17;21(1):314. doi:

10.1186/s12877-021-02240-8. PMID: 34001023; PMCID: PMC8127264.

19. Kim K-S, Park K-S, Kim M-J, Kim S-K, Cho Y-W, Park SW. Type 2 diabetes is associated with low muscle mass in older adults. Geriatr Gerontol Int. 2014;14:115-21. doi:10.1111/ggi.12189. 
20. Quah JH, Wang P, Ng RRG, Luo N, Tan NC. Health-related quality of life of older Asian patients with multimorbidity in primary care in a developed nation.Geriatrics and Gerontology International. 2017 Oct 17 (10)1429-1437.

21. R Hirschmann. Demographics of Singapore - statistics \& facts, 5, July 2021.https://www.statista.com/topics/5763/demographics-of-singapore/. Assessed on 21 Aug 2021.

22. Linton E, Gubhaju B, Chan A. Home alone: older adults in Singapore. Research Brief Series 4 report, CARE 2018/4.

23. Chan, Angelique, Chetna Malhotra, Rahul Malhotra, and Truls Ostbye. "Living arrangements, social networks, and depressive symptoms among older men and women in Singapore." Geriatric Psychiatry. 2010: 630-639.

24. Ng LP, Koh YLE, Tan NC. Physical acitivity and sedentary behavior of ambulatory older adults in a developed Asian community: a cross-sectional study. Singapore med J. 2020 May;61(5): 266-271. Doi: 10.11622/smedj.2020022.

25. Kirwan R, McCullough D, et al. Sarcopenia during COVID-19 lockdown restrictions: long term health effects of short term muscle loss. GeroScience. 2020 Dec; 42(6):1547-1578. Doi: 10.1007/s11357020-00272-3.

26. Ghram A, Briki W, Mansoor $\mathrm{H}$ et al. Home-based exercise can be beneficial for counteracting sedentary behavior and physical inactivity during the COVID-19 pandemic in older adults. Postgraduate Med 2021; 133(5). https://doi.org/10.1080/00325481.2020.1860394. 